\title{
Investigation of local work function distribution of field emission cathode from carbon materials doped by Ba with the help of AFM/SEM/X-ray
}

\author{
P.V. Sherstnev*, A.S. Baturin, V.S. Bormashov, K.N. Nikolski, E.P. Sheshin \\ Moscow Institute of Physics and Technology, Institutskij per., 9, Dolgoprudny 141700, Russia
}

\begin{abstract}
The joint application of atomic force microscopy (AFM) and SEM/X-ray micro-analyzer for investigation of electron work function (EWF) distribution over the surface of field emission cathode (FEC) is described in the paper. This method was used to investigate the graphite cathodes surface after barium doping. The possibility of defining the average value of EWF over the surface of FEC is shown. The results of AFM measurements are compared with results of field emission tests. The reduction of work function down to $3-3.2 \mathrm{eV}$ was found after barium doping.

(C) 2003 Elsevier Ltd. All rights reserved.
\end{abstract}

\section{Introduction}

The emissions properties of the surface are defined by many parameters. The most important parameters are an electron work function (EWF) and geometrical sizes of emission centers (field enhancement factor). Generally it is difficult to determine the dominant parameter influencing on appears of emission current. Simultaneous investigation of local distribution of EWF and surface topography can partially solve this problem. Early it was reported [1] about simultaneous investigation of topography, local electroconductivity and distribution of EWF over the surface with usage of scanning tunneling field emission microscope, which operates in high vacuum. With the help of this method, the position of intensive, separately situated emissions sites ('individual' emission centers) were determined over a large surface area, whereupon an electron escape and a surface electron potential at the 'individual' center were investigated in detail.

It is well know that the filed emissions cathodes (FECs) work function reduction should result in decreasing of the operating voltage. One of the possible ways of the work function's reduction consists in doping alkali, alkali-earth and rare earth metals into graphite structures [2]. The efficiency of such method could be estimated by measurements of distribution of EWF over the FECs surface before and after doping.

\footnotetext{
* Corresponding author. Fax: + 7-095-409-95-43.

E-mail address: pavel@lafeet.mipt.ru (P.V. Sherstnev).
}

The investigation of EWF over the surface of thermionic cathodes doped by barium with the help of AFM/STM (scanning tunneling microscope) and SEM/EDX is previously described [3]. However, the measurement procedure described in that article does not allow to define the absolute value of EWF. We propose to use only atomic force microscopy (AFM) and SEM/X-ray micro-analyzer for estimation of doping efficiency and correspondingly for localization of separately situated emission centers.

In the given work, the distribution of EWF was investigated for two cathodes. One of the cathodes was doped by $\mathrm{BaBr}_{2}$. Another one was undoped. At first, the topography and distribution of chemical components of FECs were investigated by SEM/X-ray micro-analyzer. Further, using the data obtained from SEM/X-ray microanalyzer, the AFM measurements were carried out in several regions containing different chemical elements. The reduction of the average EWF value of doped cathode in comparison with undoped cathode was observed. The ration of EWFs was independently estimated from field emission characteristics of given cathodes. The EWF measured by AFM are compared with results of field emission tests.

\section{AFM mode of measurement of electron work function}

The AFM investigations have been carried out with a scanning probe microscopy Solver P47 (produced by NTMDT) designed for operation in open air. The scanner investigated the maximum area to be about $50 \times 50 \mu \mathrm{m}^{2}$. 


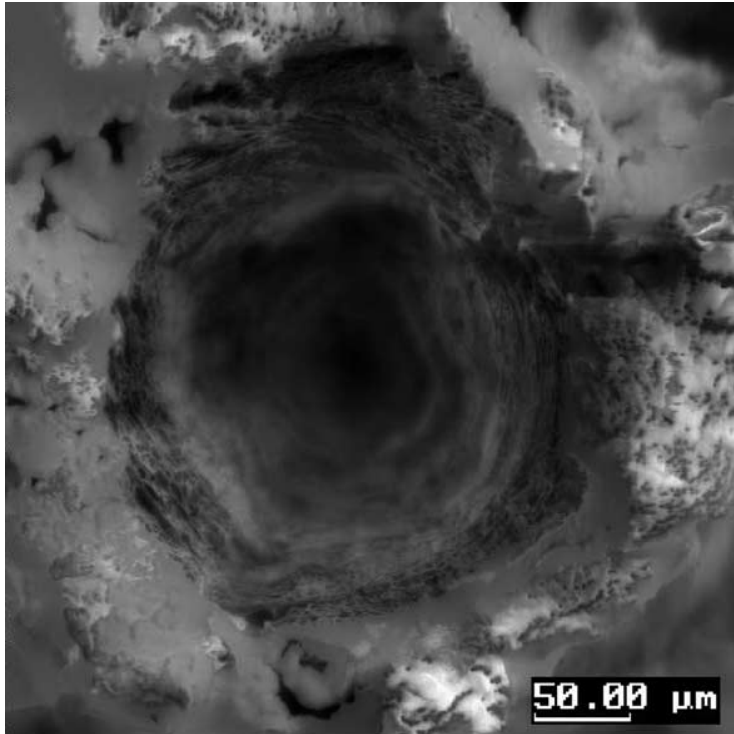

Fig. 1. SEM image of doped cathode

The minimum step of scanning a long axes $X, Y$ is $0.14 \AA$ and along axis $Z$ is $0.85 \AA$. The ranging of $Z$ scanner is about $3 \mu \mathrm{m}$. The AFM measuring of topography has been carried out in semicontact mode with $\mathrm{V}$ form silicon cantilever with radius of curvature less then $10 \mathrm{~nm}$.
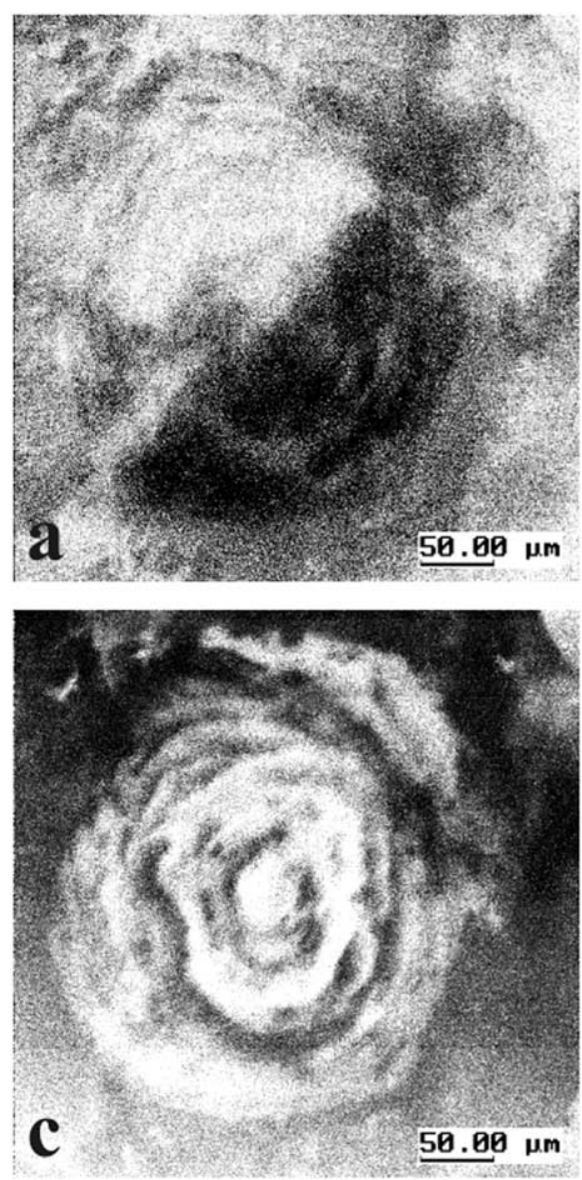

AFM allows investigation of surface topography as well as visualization of surface potential [4]. So called 'Kelvin mode' is used in AFM to determine the surface potential, which is connected with local distribution of EWF. This method consists in registration of electrically excited oscillation of cantilever under influence of the force proportional to the potential difference between cantilever and sample. The potential difference between cantilever and sample depends on difference between surface potential of sample and applied constant voltage. When the applied constant voltage is equal to the value of surface potential, the detecting signal, proportional to the amplitude of oscillation of cantilever, must be zero. The distance between sample and cantilever during scanning are supported to constant.

For estimating of EWF of sample it is necessary to know the EWF of cantilevers. For this purpose, the sample with given value of EWF must be investigated. The binary composition $\mathrm{Cu}-\mathrm{Cr}$ was used to make such calibration. This composition does not form an alloy. Its surface consists of individual grains of $\mathrm{Cr}$ and $\mathrm{Cu}$. Those grains are clearly visible in optic microscope. The scanning area of AFM allows investigating the boundary between of $\mathrm{Cu}$ and $\mathrm{Cr}$ grains, which is found with the help of optical microscope. Thus, the surface potential of two different materials are
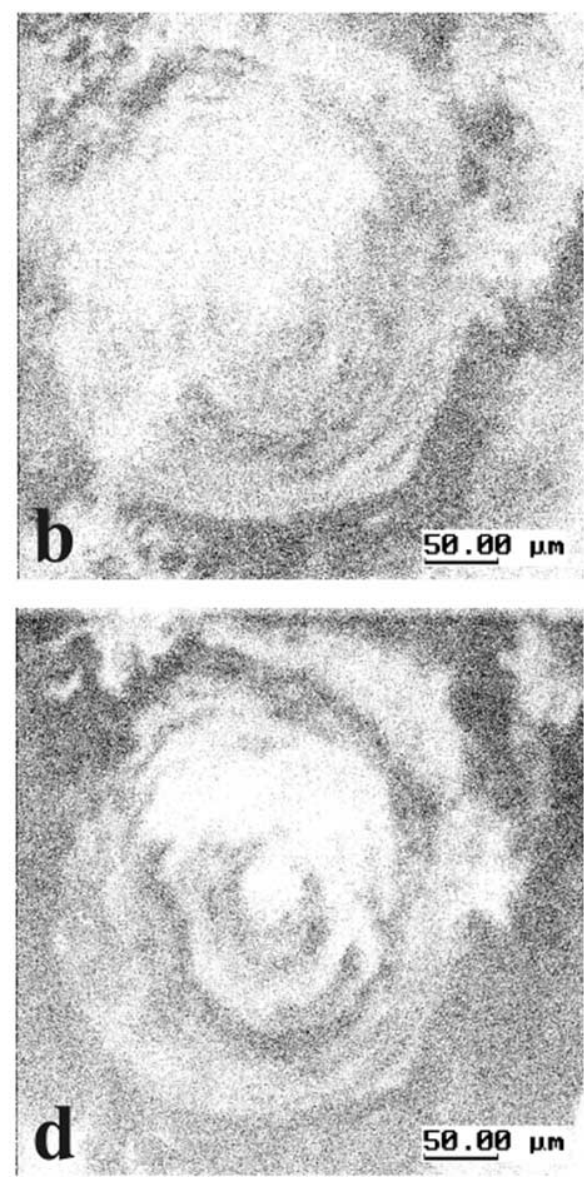

Fig. 2. Distribution of chemical elements of doped cathode (a) is carbon, (b) is oxygen, (c) is bromium, (d) is barium. 
investigated under the same conditions that permit to define more precise the EWF of cantilever.

The surface potential of sample $\mathrm{Cu}-\mathrm{Cr}$ which were measured by above described method are $\varphi_{\mathrm{Cu}} \approx-1.48 \pm$ $0.05 \mathrm{eV}, \varphi_{\mathrm{Cr}} \approx-1.32 \pm 0.05 \mathrm{eV}$. The differences of EWF is $\varphi_{\mathrm{Cr}}-\varphi_{\mathrm{Cu}}=0.16 \pm 0.05 \mathrm{eV}$. The tabular data of difference of EWF is $\left(\varphi_{\mathrm{Cr}}-\varphi_{\mathrm{Cu}}\right)_{\mathrm{tab}}=0.18 \mathrm{eV}$. Thus, by the help of AFM it is possible sufficiently precise to measured EWF of sample. According to tabular data and results obtained from AFM measuring, the EWF of cantilever must be $\varphi_{\text {cant }}=5.89 \pm 0.05 \mathrm{eV}$.

\section{Researched samples and results of measurements}

In the given work, the distribution of EWF was investigated for two cathodes prepared from exfoliated graphite. One of the cathodes was doped by $\mathrm{BaBr}_{2}$ and another without doping. The emission centers on the surfaces of exfoliated graphite were made with the help of laser. Under action of laser radiation on the surface of exfoliated graphite damages and roughness are formed. Depending on power of laser radiation it can be either craters or through apertures with jagged edges. In this work, the craters were emission centers. For the doping of cathode, the exfoliated graphite was situated in rich solution of $\mathrm{BaBr}_{2}$ during one minute. After that, the procedure of washing and annealing under the temperature $700{ }^{\circ} \mathrm{C}$ were carried out. Then, exfoliated graphite was subjected to laser treatment. After then, the samples are investigated in SEM and X-ray micro-analyzer. Figs. 1 and 2 show SEM topography and distribution of chemical elements of one doped cathode. Further, using the data obtained from SEM/X-ray microanalyzer, the AFM measurements were carried out in several regions containing different chemical elements. As since, the topography of experimental sample has a very

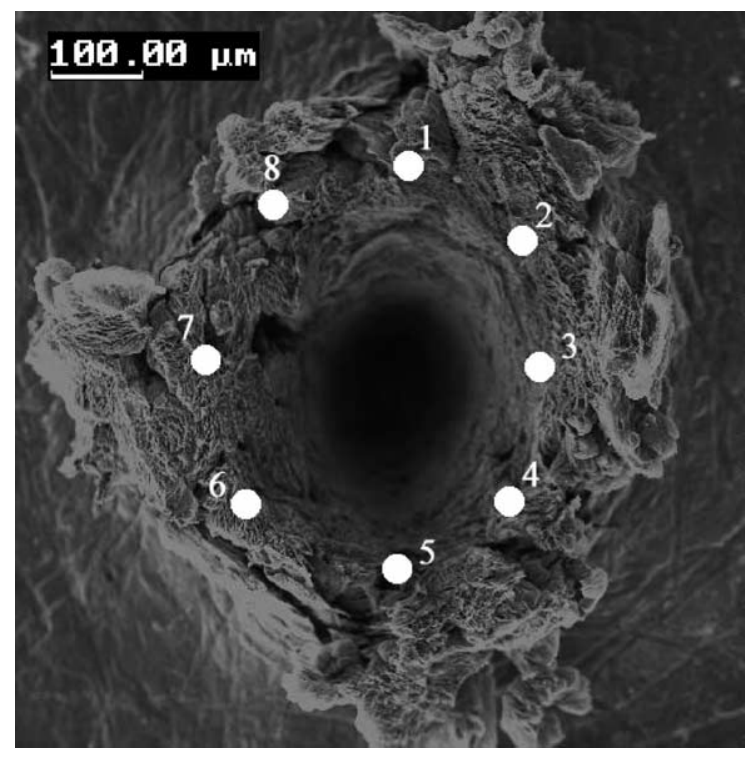

Fig. 3. SEM image of undoped cathode.

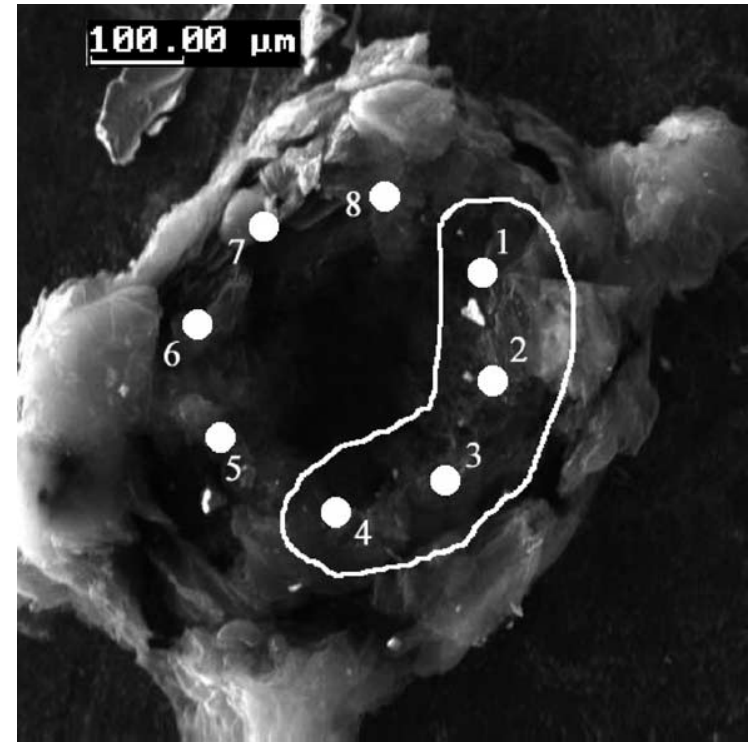

Fig. 4. SEM image another doped cathode.

shape relief, the AFM investigation were carried out in very small area of square about $1 \times 1 \mu \mathrm{m}^{2}$. Figs. 3 and 4 show the SEM images of doped and undoped cathode. The regions of AFM investigations are pointed by white dots. The results of investigation of EWF in those regions are presented in Table 1. The distributions of $\mathrm{Ba}$ and $\mathrm{Br}$ over the surfaces of doped cathode are presented in Fig. 5.

The average value of EWF of undoped sample is $(4.32 \pm 0.05) \mathrm{eV}$. For the doped sample there are two regions with a different average value of EWF $(3.05 \pm 0.05) \mathrm{eV}$ and $(4.3 \pm 0.05) \mathrm{eV}$. The areas with reduced EWF are surrounded by white line in the SEM image of doped cathode.

\section{Emission characteristics of doped and undoped cathodes}

Field emission tests of doped and undoped cathodes were performed in a diode system. The anode was made

Table 1

The value of electron work function in corresponding regions of Figs. 3 and 4

\begin{tabular}{llll}
\hline $\begin{array}{l}\text { The regions } \\
\text { number }\end{array}$ & $\begin{array}{l}\text { Electron work function } \\
\text { of undoped cathode, eV }\end{array}$ & $\begin{array}{l}\text { Electron work } \\
\text { function of doped } \\
\text { cathode, eV }\end{array}$ \\
\hline 1 & 4.32 & 3.63 & - \\
2 & 4.32 & 2.23 & - \\
3 & 4.34 & 3.38 & - \\
4 & 4.31 & 2.98 & - \\
5 & 4.28 & - & 4.3 \\
6 & 4.32 & - & 4.32 \\
7 & 4.33 & - & 4.35 \\
8 & 4.36 & - & 4.23 \\
Average value & 4.32 & 3.05 & 4.3 \\
\hline
\end{tabular}



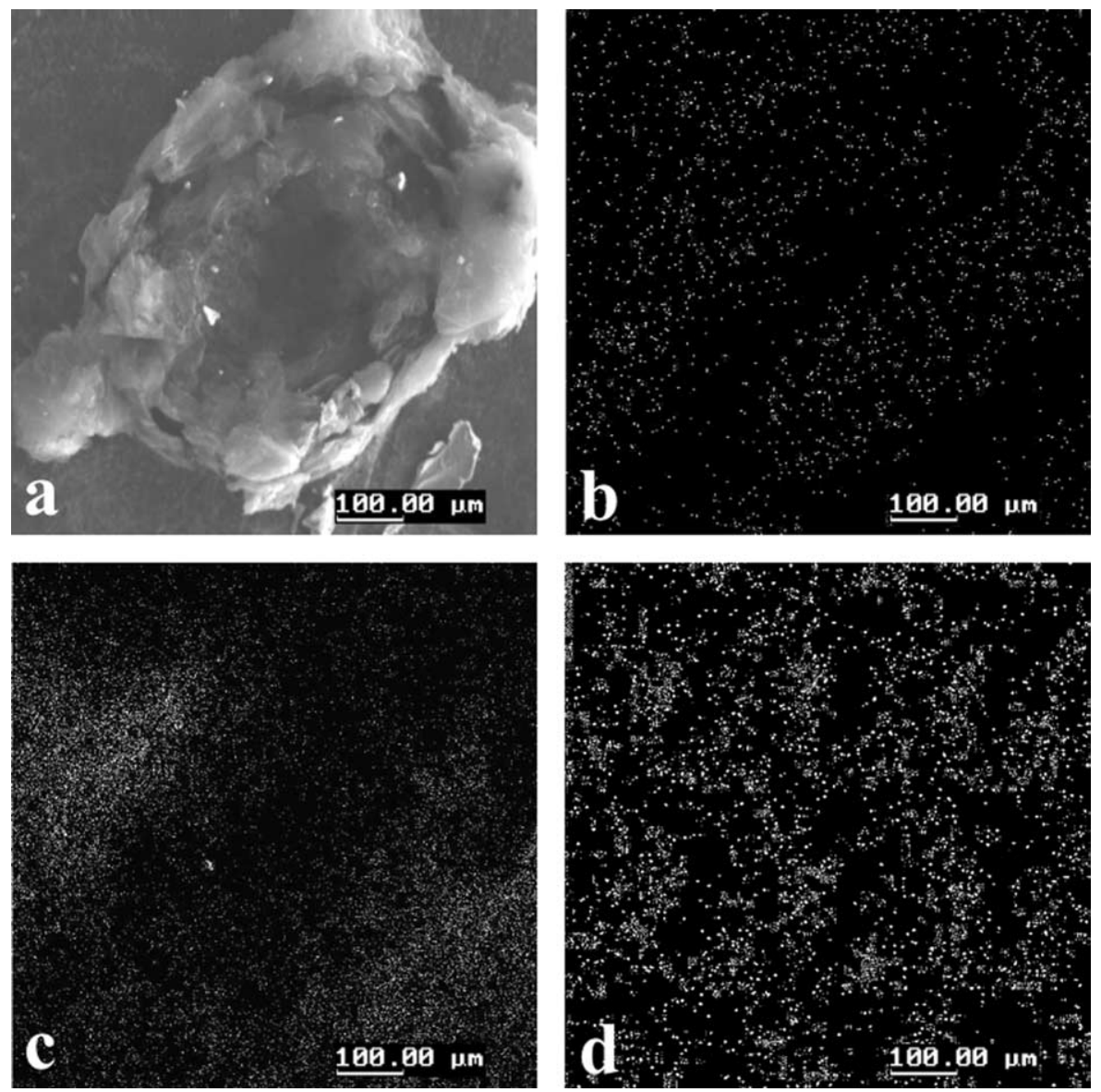

Fig. 5. Distribution of chemical elements over the surfaces of doped cathode. (a) is SEM image of cathode, (b) is oxygen, (c) is bromium, (d) is barium.

from glass plate with conductive ITO layer coated by phosphor. The cathode was glued to the anode on the spacers that define the distance between anode and cathode, which was $800 \mu \mathrm{m}$. The tests were performed in the vacuum chamber with residual gas pressure lower than $10^{-6}$ Torr.

In Fig. 6, Fowler-Nordheim curves for two types of cathodes are presented. One can conclude that emission characteristics of doped and undoped cathodes distinguish

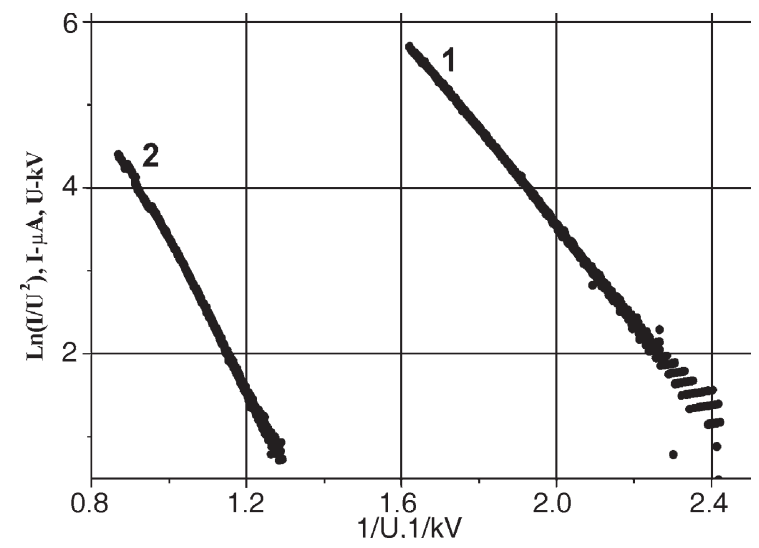

Fig. 6. Fowler-Nordheim plots of cathodes prepared from exfoliated graphite. 1 , corresponds to the doped cathode; 2, corresponds to the undoped cathode. by applied voltage and the slopes of the Fowler-Nordheim curves. Assuming, that the form factor of the cathodes are the same, then this difference can be explained by the difference of EWF of cathodes. Suggesting that the EWF of undoped cathode is $4.3 \mathrm{eV}$ one can find the experimental estimation for the EWF of doped cathode is $3.2 \mathrm{eV}$.

Thus, the doping of emission cathodes by $\mathrm{BaBr}_{2}$ is the effective method to decrease an EWF and correspondingly decrease an extracting voltage.

\section{Conclusion}

New method of investigation of EWF with the help of SEM/X-ray micro-analyzer and AFM are suggested.

The average meanings of EWF of doped exfoliated graphite cathode measuring by AFM coincide with the meaning of EWF calculating from emission characteristics of cathodes. Thus, AFM allows sufficiently simply and exactly to measure the distribution of EWF over the surface of cathode and correspondingly predict the place of emissions centers.

AFM is convenient instrument for estimation of efficient doping of cathodes by different alkali, alkali-earth and rare earth metals. 


\section{References}

[1] V.D. Frolov, A.V. Karabutov, S.M. Pimenov, V.I. Konov, Electronic properties of the emission sites of low-field emitting diamond films, Diamond and Related Materials 9 (2000) 1196-1200.

[2] A.S. Baturin, K.N. Nikolski, E.P. Sheshin, R.G. Tchesov, B.S. Borisov, E.P. Kirilenko, A.Yu. Trifonov, Alkali and rare earth metal doping of carbon materials to improve their field emission properties, Technical Digest, IVESC 2000, p. 52.

[3] C. Makovicka, G. Gartner, A. Hardt, W. Hermann, D.U. Wiechert, Impregnated cathode surface investigations by SFM/STM and SEM/ EDX, Applied Surface Science 111 (1997) 70-75.

[4] M. Nonnenmacher, M.P. O’Boyle, H.K. Wickramasinghe, Applied Physics Letters 58 (1991) 2921. 